\title{
Length illusions in conventional and single-wing Müller-Lyer stimuli
}

\author{
JOHN PREDEBON \\ University of Sydney, Sydney, New South Wales, Australia
}

\begin{abstract}
Warren and Bashford (1977) reported that eliminating one of the wing components from the conventional (i.e., two-wing) Müller-Lyer figures had no appreciable effect on the magnitude of the acuteangle (contraction) illusion but substantially reduced the magnitude of the obtuse-angle (expansion) illusion. In addition, they found that whereas the contractionary effects of the acute-angle components tended to be confined to the region of the shaft adjacent to the angles, the expansionary effects of the obtuse-angle components were more uniformly distributed across the shaft. Since these findings challenge many theories of the Müller-Lyer illusion, the purpose of the present investigation was to evaluate further Warren and Bashford's work with four experiments. Experiments 1 and 2 assessed length illusion magnitudes by requiring subjects to adjust either the length of a plain comparison line to match the length of the Müller-Lyer test figures (Experiment 1) or the length of comparison Müller-Lyer figures to match the length of plain test lines (Experiment 2). Experiments 3 and 4 used a bisection task to assess whether the illusory effects of the angle components are confined mainly to regions of the shaft adjacent to the angles. Consistent with most theories of the Müller-Lyer illusion, eliminating one of the wing components reduced both forms of the Müller-Lyer length illusion to a similar extent. In addition, the acute- and obtuse-angle forms yielded similar patterns of bisection errors, with substantial errors for regions of the shaft adjacent to the angles and negligible errors for regions of the shaft distant from the angles.
\end{abstract}

The Müller-Lyer illusion is one of the best-known geometrical illusions. In its conventional or classic form (Müller-Lyer, 1889), the illusion consists of the acuteangle illusion of shaft underestimation and the obtuseangle illusion of shaft overestimation (see Figure 1A). The illusion has been researched extensively, partly because it has many variations (Restle \& Decker, 1977) and partly because of the long-standing belief that the study of visual illusions, such as the Müller-Lyer illusion, is capable of revealing the principles governing nonillusory visual perception (Warren, 1976; Warren \& Bashford, 1977).

Many theories of vision have been offered to explain the classic Müller-Lyer illusion, including direct size scaling (Gillam, 1998; Gregory, 1963), perceptual assimilation (Brigell, Uhlarik, \& Goldhorn, 1977; Day \& Dickinson, 1976; Pressey \& Pressey, 1992) of the length of the shaft toward the lengths of contextual elements, incorrect encoding of the positions of the vertices of the wings (Chiang, 1968; Glennerster \& Rogers, 1993; Morgan, Hole, \& Glennerster, 1990; Stuart, Day, \& Dickinson, 1984), properties of the low spatial frequency visual channels (Ginsburg, 1984), and object recognition processes, such as mechanisms associated with preperceptual adjust-

I am grateful to D. J. Aks, S. Coren, R. M. Warren, and an anonymous reviewer for their helpful comments on an earlier version of the paper. Correspondence concerning this article should be addressed to J. Predebon, Department of Psychology, University of Sydney, Sydney, NSW 2006, Australia (e-mail: johnp@psych.usyd.edu.au). ments (Warren \& Bashford, 1977) and visual scene interpretation (Redding \& Hawley, 1993). Importantly, although the theories differ in the nature of the postulated illusion-inducing mechanism or process, all assume that similar mechanisms are implicated in both the over- and the underestimated Müller-Lyer forms.

However, the results of Warren and Bashford's (1977) important (although relatively neglected) study, which assessed perceptual size distortions in a variety of Müller-Lyer figures, pose a major challenge to the assumption that the two Müller-Lyer illusion forms are mediated by the same mechanism. Two aspects of their findings are relevant to this issue. First, Warren and Bashford found that reducing the classic figures (Figure 1A) to their one-wing versions (see Figures $1 \mathrm{~B}$ and $\mathrm{IC}$ ) substantially decreased the magnitude of the obtuse-angle length illusion, while leaving the magnitude of the acute-angle length illusion virtually unaffected. Second, on the basis of the results of a novel procedure that required subjects to partition the shaft of the figures into subjectively equal quarters, they reported a substantial difference in the range of influence, or scaling effect, of the acute- and obtuseangle components on the adjacent space in the classic Müller-Lyer forms and, to a lesser extent, in the corresponding one-wing versions. Specifically, whereas the influence of the angle components in the acute-angle (underestimated) form was considerably greater for the portion of the shaft adjacent to the angle, the illusory effect of the angles in the obtuse-angle (overestimated) form tended to be more uniformly distributed over the 


\begin{abstract}
obtuse-angle
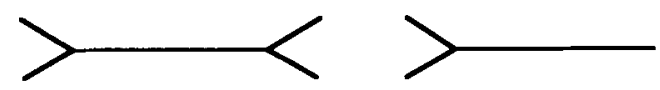

control

acute-angle

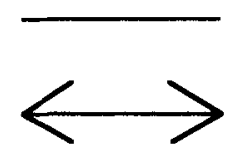

A (both wings)

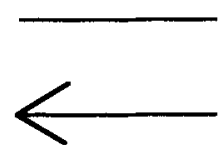

B (left wing)
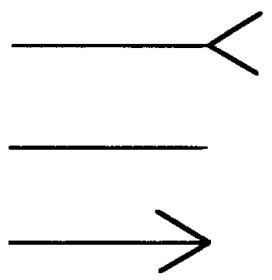

$\mathrm{C}$ (right wing)

Figure 1. The test figures used in Experiment 1 and the comparison figures used in Experiment 2 . In each experiment, independent groups of subjects were given the $A, B$, or $C$ figures. The $A$ figures are the two-wing, or conventional, acute-angle (underestimated) and obtuse-angle (overestimated) forms of the Müller-Lyer illusion, and the $B$ and $C$ figures are the corresponding one-wing versions.
\end{abstract}

entire length of the shaft (Warren \& Bashford, 1977, p. 621).

Both sets of findings impose major constraints on the main theories of the illusion, except for the object recognition theories (Redding \& Hawley, 1993; Warren \& Bashford, 1977), which, in any case, were prompted by these findings. For example, the differential effect of eliminating one of the wing components on the illusion magnitudes of the under- and overestimated Müller-Lyer forms is difficult to reconcile with assimilation theories (e.g., Day \& Dickinson, 1976). According to these theories, eliminating an angle component from the conventional figure effects a reduction in the number of illusion-inducing contextual elements (i.e., wings), thereby resulting in a decrease in the magnitudes of both the acute- and the obtuse-angle illusions. Similar effects are predicted by the positionencoding theories (Glennerster \& Rogers, 1993; Morgan et al., 1990). These theories assume that the neural computations of the locations of the shaft's endpoints are influenced by the wings of the angle components, the effect of which is to displace their positions in the neural image toward each other for the acute-angle form and away from each other for the obtuse-angle form, which in turn produces the apparent expansion and contraction, respectively, of the shaft's extent. Since only one of the shaft's endpoints (i.e., the angle end) is encoded incorrectly in the one-wing figures, both the acute- and the obtuseangle forms should yield substantially smaller illusions than do the corresponding conventional forms. Thus, neither the assimilation nor the position-encoding theories are consistent with Warren and Bashford's finding of a substantial reduction in the magnitudes of the obtuseangle but not of the acute-angle one-wing length illusions.

Warren and Bashford's (1977) other major finding, indicating a different scaling effect of the acute and the obtuse angle components, also raises problems for many theories of the illusion. In general, the theories predict similar scaling effects of the acute- and the obtuse-angle components, although they differ with respect to the spatial range of the angles' influences. For example, some theories (e.g., position encoding) imply that the range of illusory influence of the angle components in both forms of the Müller-
Lyer illusion is restricted to the region of the shaft adjacent to the angle, whereas other theories (e.g., assimilation, size scaling) imply a more generalized effect over the entire length of the shaft.

In view of the major implications of Warren and Bashford's (1977) work for many of the main theories of the Müller-Lyer illusion, the purpose of the present investigation was to examine further the pattern of illusory effects in various versions of the acute- and obtuse-angle Müller-Lyer forms. Two additional reasons motivated the investigation. First, the significance of Warren and Bashford's findings for theory needs to be qualified by two possible limitations of their work. (1) There was an absence of appropriate control conditions (i.e., length judgments of plain lines), which prohibits an unambiguous assessment of the relative illusion magnitudes across the different Müller-Lyer conditions in their experiments. (2) They did not report statistical analyses to establish the reliability of the scaling effects of the angle components.

Second, the results of other studies have not been consistent with Warren and Bashford's (1977) work. Although performed in a different context, three studies (Day \& Dickinson, 1976; Predebon, 1994, 1996) reported a substantial reduction from the conventional illusion in the magnitudes of both forms of the one-wing illusions. Redding and Hawley (1993) also found reductions in the magnitudes of both one-wing illusions but claimed that their data showed a greater reduction for the obtuse-angle form; unfortunately, they did not report any statistical tests for the latter claim. Similarly, Warren and Bashford's finding of a differential scaling effect of the acute and the obtuse angles appears to be inconsistent with Morinaga's (in Oyama, 1960) work, which indicates that the illusory effects are restricted to the region of the shaft adjacent to the wings. However, as Oyama did not specifically refer to the distinction between the acute- and the obtuse-angle forms, it is uncertain whether his summary of Morinaga's work refers to the obtuse-angle form, to the acute-angle form, or to both forms.

In the four experiments described below, two different illusory effects were investigated: length illusion magnitudes (Experiments 1 and 2) and the scaling, or spatial range of influence (Experiments 3 and 4), of the angles on 
the shaft's apparent length. These effects, of course, need not be related. For example, demonstrating an equivalence in the pattern of length illusion magnitudes across the two Müller-Lyer forms (and their one-wing versions) does not entail the conclusion of equivalent scaling effects of the acute and obtuse angles. Similarly, demonstrating an equivalence in the scaling effects (spatial range of influence) of the acute- and obtuse-angle components does not imply an equivalence in the absolute illusion magnitudes of the two Müller-Lyer forms. Both effects, therefore, were investigated, especially since Warren and Bashford (1977) based their conclusion of a fundamental difference between the acute- and the obtuse-angle Müller-Lyer forms on their investigations of both illusory effects.

The magnitudes of the one-wing and the two-wing (i.e., conventional) length illusions were quantified in Experiments 1 and 2 by requiring subjects to adjust the length of either a plain comparison line (Experiment 1) or Müller-Lyer comparison figures (Experiment 2) to match the apparent length of the test figures. Taken together, the results of these two experiments were expected to provide converging evidence for the pattern of length illusion magnitudes across the acute- and obtuse-angle Müller-Lyer forms. Assessment of the scaling effects of the angle components required a different methodology. Experiments 3 and 4, therefore, used Warren and Bashford's (1977) bisection task to map the spatial range of influence of the angle components on the shaft's apparent length. The bisection task required subjects to indicate the apparent midpoint of either the shaft (Experiment 4) or segments of the shaft (Experiments 3 and 4).

The critical issue of interest was the similarity or otherwise of the patterns - not the absolute magnitudesof illusory effects produced by the acute- and obtuseangle Müller-Lyer stimulus conditions. This issue was evaluated statistically by assessing the relevant interaction in an analysis of variance (ANOVA) performed on the scaled length-matching data of Experiments 1 and 2 and the corresponding interaction in an ANOVA performed on the scaled bisection data of Experiments 3 and 4 .

\section{EXPERIMENT 1}

To facilitate comparison with the results of previous studies, the design and stimuli of Experiment 1 were similar to those of Redding and Hawley's (1993) experiments, with one main exception: Whereas they measured illusion magnitude by requiring subjects to demarcate the length of segment on a plain response that matched the apparent length of the shaft, Experiment 1 quantified the illusory effects by using the conventional method of adjustment. ${ }^{1}$ As in Redding and Hawley's experiments, independent groups of subjects were allocated to each of the three stimulus sets shown in Figure 1.

\section{Method}

Subjects. The subjects were 42 volunteer undergraduate students recruited from an introductory course in psychology. All had normal or corrected-to-normal vision.
Stimuli and Apparatus. There were three sets of stimuli, each consisting of three types of figures: a plain control line and the acute- and the obtuse-angle Müller-Lyer forms. The three sets differed in the number and position of the wing components: at both ends, at the left end only, or at the right end only (see Figures l A, $1 \mathrm{~B}$, and $1 \mathrm{C}$, respectively). There were two versions for each set of three figures, one with $50-\mathrm{mm}$-long and one with $100-\mathrm{mm}-\mathrm{long}$ horizontal lines. Each figure was centered on a sheet of white paper $21 \times 29.5 \mathrm{~cm}$, with the horizontal line drawn parallel to the long axis of the sheet. All the lines were $0.5 \mathrm{~mm}$ wide. The wings formed an internal angle of $60^{\circ}$, the lengths of the fins were 15 and $30 \mathrm{~mm}$ for the 50- and 100-mm-long Müller-Lyer figures, respectively. The lengths of the fins, which were a constant fraction (.3) of shaft length, were selected to maximize the magnitudes of the illusory effects. ${ }^{2}$

The test figure was attached to a large white stimulus surface, which was tilted back from vertical. This surface formed an angle of $135^{\circ}$ with the horizontal surface of the comparison device. The comparison device consisted of an adjustable white opaque slide and a white display area, on which was drawn a $0.5-\mathrm{mm}$-wide horizontal comparison line. The slide was positioned between guides and rested on the display surface. The subject could move the slide laterally to cover or uncover the comparison line. A scale attached to one of the guides enabled measurements of the comparison line settings to the nearest $0.5 \mathrm{~mm}$. Both the guides and the scale were occluded from the subject's view. The subjects viewed both the stimulus figures and the comparison line from a distance of approximately $50 \mathrm{~cm}$. From this distance, the 50 - and $100-\mathrm{mm}$ stimulus figures subtended visual angles of approximately $5.7^{\circ}$ and $11.4^{\circ}$, respectively.

Design and Procedure. Wing position was the between-subjects factor, and type of figure and length of shaft were the within-subjects factors. The subjects were allocated in order of arrival to one of the three wing position conditions, with 14 subjects in each condition.

The 50 - and $100-\mathrm{mm}$ stimuli of each set were presented separately in two blocks of trials, with half the subjects first presented with the $100-\mathrm{mm}$ stimuli and the other half with the $50-\mathrm{mm}$ stimuli. The order of presentation of the three figures within each block was randomized. There were four adjustments for each stimulus, two with the comparison line set initially to an extent that was clearly longer (L) and two with the comparison line set initially to an extent that was clearly shorter (S) than the stimulus figure. Half the subjects always made the four adjustments for all stimulus figures in the order LSSL, and the other half in the order SLLS, a total of 24 adjustments for each subject. The subjects completed the four adjustments for each test figure before proceeding to the next test figure. The instructions required the subjects to adjust the length of the comparison line so that it appeared to be equal to the length of the horizontal line of the stimulus figure.

\section{Results}

The four length adjustments for each stimulus figure were averaged to give six scores ( 3 figures $\times 2$ lengths) for each subject. The results of the 50 - and $100-\mathrm{mm}$ conditions are shown in Figure 2.

The 3 (figure) $\times 3$ (wing position) mixed design ANOVA performed separately on the length-matching data of the 50- and 100-mm stimulus conditions produced a nonsignificant main effect of wing position $[F(2,39)=$ 2.81 and $1.34, p>.05$, respectively] and a significant main effect of figure $[F(2,78)=243.87$ and $171.89, p<$ .05 , respectively]. The latter outcome merely confirms the occurrence of the usual Müller-Lyer effect, under- and overestimation of the acute- and obtuse-angle MüllerLyer forms, respectively. For both the 50- and the $100-$ $\mathrm{mm}$ stimuli, the figure $\times$ wing position interaction was significant $[F(4,78)=16.45$ and $14.51, p<.05$, respec- 
A

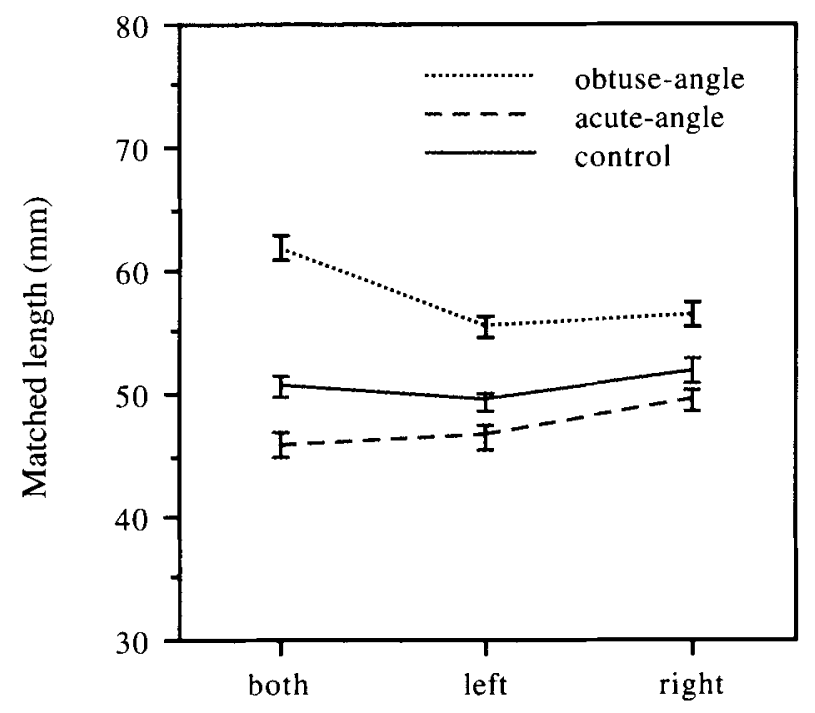

Position of wings

B

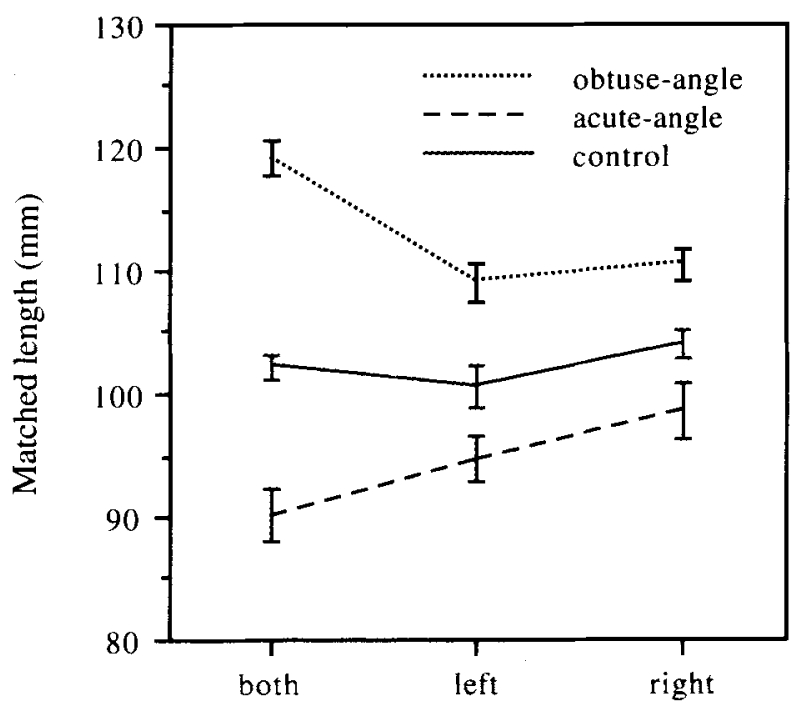

Position of wings

Figure 2. Experiment 1: Means in millimeters of the length adjustments of a plain horizontal comparison line to the apparent length of the horizontal line of the test figure as a function of type of test figure (acute angle, obtuse angle, or no angle control) and wing position (both ends, left end, or right end) in the MüllerLyer test figures. Panels $A$ and $B$ are the data sets of the 50- and $100-\mathrm{mm}$ test line conditions, respectively. Vertical bars indicate $1 S E$.

tively], indicating, as is evident from Figure 2, a reduction in both the single-wing acute- and the obtuse-angle illusion magnitudes, relative to the conventional illusion magnitudes.
The significant interaction is virtually guaranteed by the opposing directional changes in the length matches of the two illusion forms. Specifically, as is evident from Figure 2, relative to the conventional illusion, the length matches were shorter for the one-wing obtuse-angle figures and longer for the one-wing acute-angle figures. Assessing the differential reduction (if any) of the acute- and obtuse-angle single-wing illusion magnitudes, therefore, requires factoring out these opposing directional trends. To this end, the length matches were scored in the first instance as deviations from the point of subjective equality (PSE). Estimates of the PSE were provided by the length matches of the control lines. Each subject's PSE for the 50and $100-\mathrm{mm}$ control lines were subtracted from the length matches of the 50- and 100-mm Müller-Lyer figures, respectively, and the results are shown in Table 1. Positive and negative errors indicate over- and underestimation, respectively, of the length of the Müller-Lyer shaft. In the second instance, the signs of all the deviation scores of the acute-angle conditions were reversed to produce scores on the same scale as the obtuse-angle deviation scores. The scaled scores thus permit an assessment of the similarity or difference in the patterns of illusion magnitudes across the acute- and obtuse-angle conditions.

The results of the 2 (figure) $\times 3$ (wing-position) repeated measures ANOVA performed on the $50-\mathrm{mm}$ scaled data set showed a significant main effect of wing position $[F(2,39)=18.45, p<.05]$; averaged across the figure factor, the means of the conventional, left, and right figure conditions were $7.49,4.48$, and $3.47 \mathrm{~mm}$, respectively. The main effect of figure also was significant $[F(1,39)=34.19, p<.05]$, indicating that, averaged across the wing position factor, the mean error was greater for the obtuse-angle than for the acute-angle conditions ( $M=6.97$ and $3.34 \mathrm{~mm}$, respectively). Importantly, the figure $\times$ wing position interaction was not significant $[F(2,39)=2.03, p>.05]$, indicating that eliminating a wing component had similar effects on the magnitudes of the acute- and the obtuse-angle illusions. The ANOVA performed on the $100-\mathrm{mm}$ scaled data set produced similar outcomes. Averaged across wing position, the illusion was greater for the obtuse- than for the acute-angle figures ( $M=10.64$ and $7.83 \mathrm{~mm}$, respectively), although the difference was marginally nonsignificant $[F(1,39)=4.05$, $p=.051]$. The main effect of wing position was significant $[F(2,39)=21.17, p<.05]$, indicating a difference between the means of the conventional, left, and right figure conditions $(M=14.53,7.23$, and $5.94 \mathrm{~mm}$, respectively). The figure $\times$ wing position interaction was not significant $[F(2,39)=0.58, p>.05]$.

\section{Discussion}

The nonsignificant interaction in the ANOVA performed on the scaled data sets indicates that reducing the conventional Müller-Lyer figures to their one-wing versions had similar effects on the acute- and the obtuseangle illusion magnitudes. In contrast, Redding and Hawley (1993) argued that their findings, which were 
Table 1

Means and Standard Errors (in Millimeters) of the Length Matches,

Expressed as Deviations from the PSE, for the Shafts of the Müller-Lyer

Test Figures as a Function of the Length of the Shaft, the Position of the Wings in the Test Figure, and the Type of Test Figure in Experiment 1

\begin{tabular}{|c|c|c|c|c|c|c|c|c|c|c|c|c|}
\hline \multirow[b]{4}{*}{ Test Figure Type } & \multicolumn{12}{|c|}{ Angles in Test Figure } \\
\hline & \multicolumn{6}{|c|}{ 50-mm Shaft } & \multicolumn{6}{|c|}{ 100-mm Shaft } \\
\hline & \multicolumn{2}{|c|}{ Both } & \multicolumn{2}{|c|}{ Left } & \multicolumn{2}{|c|}{ Right } & \multicolumn{2}{|c|}{ Both } & \multicolumn{2}{|c|}{ Left } & \multicolumn{2}{|c|}{ Right } \\
\hline & $M$ & $S E$ & $M$ & $S E$ & $M$ & $S E$ & $M$ & $S E$ & $M$ & $S E$ & $M$ & $S E$ \\
\hline Acute angle & -4.84 & 0.78 & -2.85 & 0.55 & -2.32 & 0.61 & -12.15 & 1.97 & -5.97 & 1.26 & -5.38 & 1.99 \\
\hline Obtuse angle & 10.15 & 0.76 & 6.12 & 0.77 & 4.64 & 0.84 & 16.92 & 1.33 & 8.49 & 1.33 & 6.50 & 1.35 \\
\hline
\end{tabular}

obtained using a design similar to that in Experiment 1, implied a greater reduction in illusion magnitude for the obtuse- than for the acute-angle forms, although no statistical tests were given to substantiate their claim. The apparent discrepancy between the present conclusions and Redding and Hawley's interpretation of their results, however, may relate more to differences in the level of analyses than to outright empirical inconsistencies. Conceivably, Redding and Hawley might have anticipated the present conclusions had they performed their analyses on the scaled deviation scores. Nevertheless, additional converging evidence for the conclusions derived from the present results seemed warranted. The purpose of Experiment 2 , therefore, was to assess the relative magnitudes of the conventional and single-wing illusion magnitudes, using the novel procedure of requiring subjects to adjust the length of comparison Müller-Lyer figures to match the apparent length of a plain test line.

The pattern of result in Experiment 2 was expected to be the opposite of that obtained in Experiment 1. For example, in adjusting the length of the obtuse-angle conventional figure to match the length of a plain test line, the illusory lengthening of the shaft's length must be compensated for by setting its physical length to a shorter extent than the test line. Hence, whereas in Experiment 1 the length adjustments of a plain comparison line were shorter and longer, respectively, than the actual lengths of the conventional acute- and obtuse-angle test figures, in Experiment 2 the length adjustments of the acute- and obtuseangle comparison figures were expected to be longer and shorter, respectively, than the actual length of the test line.

\section{EXPERIMENT 2}

\section{Method}

The test stimuli were 50 - and 100 -mm-long horizontal lines. Each line was centered on a sheet of white paper $21 \times 29.5 \mathrm{~cm}$. The three sets of comparison or adjustable stimuli were similar to the test stimuli used in Experiment 1 (see Figure 1). For each set, there were two versions of the Müller-Lyer comparison figures, one with wing lengths of $15 \mathrm{~mm}$ and the other with wing lengths of $30 \mathrm{~mm}$. The wings formed an internal angle of $60^{\circ}$, and all the lines of the figures were approximately $0.5 \mathrm{~mm}$ wide.

The comparison device consisted of two overlapping transparent slides. One slide was fixed to the white surface of the device, and the other was movable. The left and right components of the comparison figure were drawn on the fixed and movable slides, respec- tively. For example, for the conventional Müller-Lyer comparison figures, the left wing and a part of the contiguous shaft were drawn on the fixed slide, whereas the right wing and a part of the contiguous shaft were drawn on the movable slide. By adjusting the lateral position of the movable slide, the subject could vary the length of the (overlapping) horizontal line of the comparison figure to match the apparent length of the plain test line.

The design was identical to that in Experiment 1, except that the figure and wing position factors referred to manipulations of the Müller-Lyer comparison stimuli. The subjects were 48 undergraduate students, none of whom had participated in Experiment 1. The subjects were randomly allocated to one of the three comparison wing position conditions, with 16 subjects in each condition. Half the subjects first completed all 12 adjustments (4 adjustments $\times 3$ comparison figures) for the $50-\mathrm{mm}$ test line, followed by the 12 adjustments for the $100-\mathrm{mm}$ test line, with the order being reversed for the other half. In all other respects, the method of Experiment 2 was identical to that of Experiment 1.

\section{Results}

The data treatment and data analyses were identical to those of Experiment 1 . The means and standard errors in millimeters of the length matches for the 50 - and $100-\mathrm{mm}$ test lines are shown separately in Figure 3 . The ordering of the three curves in Figure 3 are the reverse of those in Experiment 1 (see Figure 2), for reasons described earlier.

The 3 (figure) $\times 3$ (wing position) mixed design ANOVA performed separately on the data of the 50 - and the $100-\mathrm{mm}$ test line conditions yielded a nonsignificant main effect of wing position $[F(2,45)=0.55$ and 0.03 , $p>.05$, respectively] and a significant main effect of figure $[F(2,78)=551.66$ and $396.00, p<.05$, respectively $]$, confirming the occurrence of the usual Müller-Lyer effect. The figure $\times$ wing position interaction also was significant for both the 50 -and the 100 -mm data sets $[F(4,78)=$ 33.62 and $22.71, p<.05$, respectively].

The length matches of the test lines made with the plain comparison lines provided an estimate of each subject's PSE for the 50- and 100-mm test lines. The PSE was subtracted from the length matches made with the MüllerLyer comparison figures, and the results are shown in Table 2. The sign of the scores are opposite to those in Table 1 and have opposite meaning; negative and positive scores indicate over- and underestimation, respectively, of the lengths of the shafts of the comparison figures.

As for Experiment 1, the signs of the all the deviation scores for the acute-angle conditions were reversed to produce deviation scores on the same scale as the obtuse- 
A

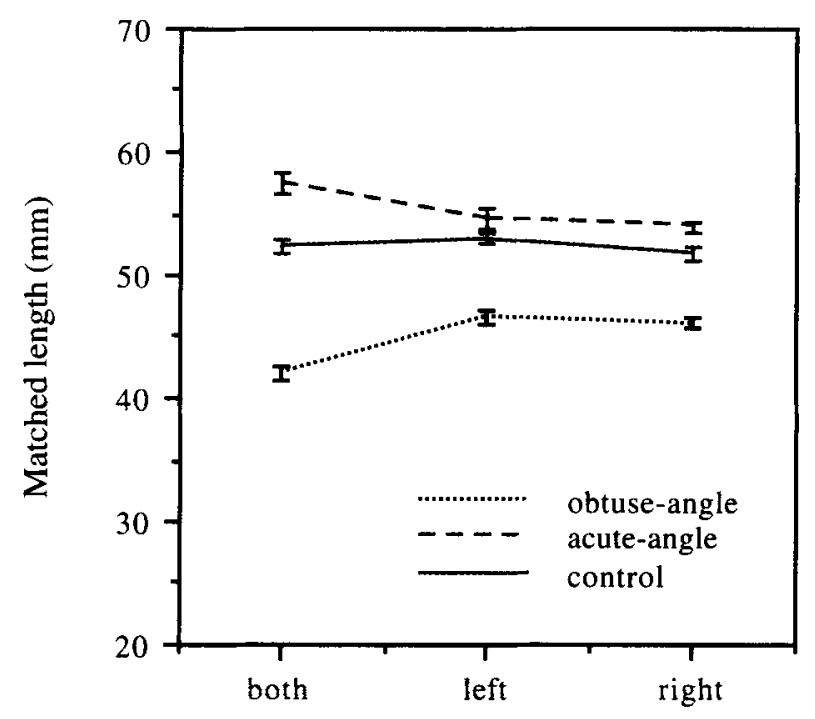

Position of wings

B

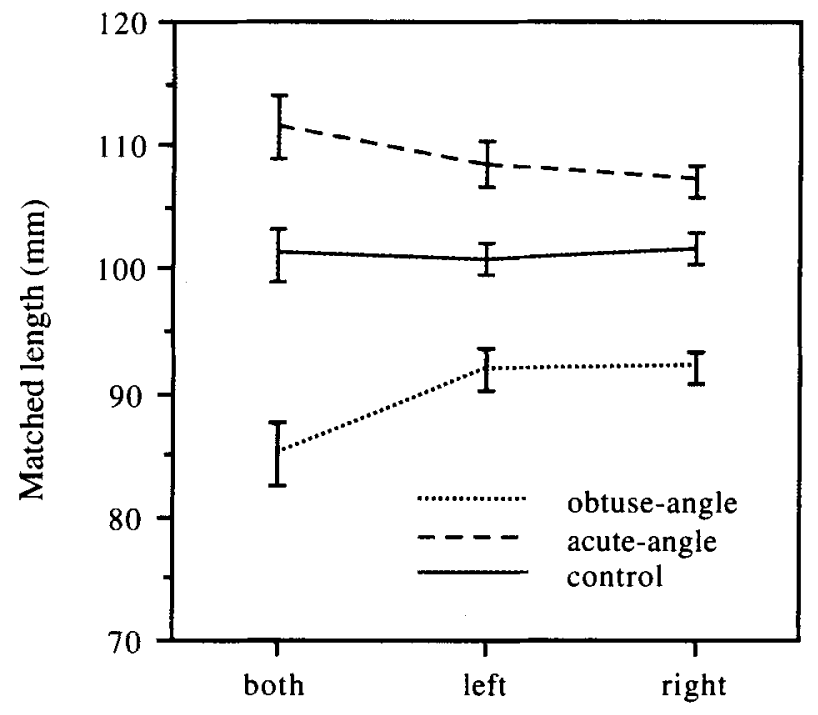

Position of wings

Figure 3. Experiment 2: Means in millimeters of the length adjustments of the horizontal line of the comparison figures to the apparent length of a plain horizontal test line as a function of type of comparison figure (acute angle, obtuse angle, or no angle control) and wing position (both ends, left end, or right end) in the Müller-Lyer comparison figures. Panels $A$ and $B$ are the data sets of the 50- and 100-mm horizontal line comparison figure conditions, respectively. Vertical bars indicate $1 S E$.

angle deviation scores. The 2 (figure) $\times 3$ (wing position) repeated measures ANOVA performed on the $50-\mathrm{mm}$ scaled data set produced a significant main effect of wing position $[F(2,45)=33.62, p<.05]$; averaged across the figure factor, the means of the conventional (two-wing) and the left and right single-wing conditions were 7.76, 3.95 , and $3.88 \mathrm{~mm}$, respectively. The main effect of figure was significant $[F(2,45)=57.08, p<.05]$, reflecting the greater mean error for the obtuse- than for the acuteangle conditions ( $M=7.47$ and $2.93 \mathrm{~mm}$, respectively). The figure $X$ wing position interaction was not significant $[F(2,45)=0.55, p>.05]$, indicating that the acuteand obtuse-angle conditions yielded similar patterns of errors across the wing position factor. The ANOVA performed on the $100-\mathrm{mm}$ scaled data set produced identical outcomes. Averaged across the wing position factor, illusion magnitude was significantly $[F(1,45)=4.716, p<$ .05 ] greater for the obtuse- than for the acute-angle figures ( $M=11.47$ and $9.29 \mathrm{~mm}$, respectively). The main effect of wing position was significant, indicating a difference between the means of the conventional (two-wing), the left, and the right single-wing conditions $(M=15.33,8.27$, and $7.53 \mathrm{~mm}$, respectively), and the figure $X$ wing position interaction was not significant $[F(2,45)=.77, p<.05]$.

\section{Discussion}

The results of Experiment 2 provide converging evidence for the findings of Experiment 1. Both show that removing one of the wing components from the conventional Müller-Lyer figures reduces the acute- and obtuseangle illusion magnitudes to a similar extent. These findings, therefore, are inconsistent with those of Warren and Bashford (1977) and with Redding and Hawley's (1993) interpretation of their own data.

The results of Experiments 1 and 2 are consistent with many theories of the Müller-Lyer illusion. As was noted earlier, however, the theories predict not only a reduction in the magnitudes of both forms of the single-wing illusions but also similar scaling effects of the acute and obtuse angles. The data of Experiments 1 and 2 are neutral with respect to the latter issue; they are consistent with either similar or different scaling effects. It is necessary, therefore, to establish the spatial range of influence of the acute and obtuse angles on the shaft's apparent length, especially since the evidence on this issue is equivocal, with Morinaga's (in Oyama, 1960) work indicating a similarity and Warren and Bashford (1977) claiming a difference in the scaling effects of acute and obtuse angles. Experiment 3, therefore, measured the scaling effect of the angle component in the single-wing versions of the acute- and obtuse-angle Müller-Lyer forms, using a version of Warren and Bashford's bisection task of fractionating the shafts into subjectively equal quarters.

\section{EXPERIMENT 3}

Experiment 3 assessed the scaling influence of the angle component in single-wing figures on segments of the shaft adjacent to and distant from the angle. As is shown in Figure 4, a small vertical mark demarcated the shafts into two equally long extents (hereafter, the segment contiguous to the angle is referred to as the angle extent, and the other as the no-angle extent), and the sub- 
Table 2

Means and Standard Errors (in Millimeters) Expressed as Deviations

From PSE, of the Length Matches for 50- and 100-mm-Long Test Lines

as a Function of the Position of the Wings in the Comparison Figure and the Type of Comparison Figure in Experiment 2

\begin{tabular}{|c|c|c|c|c|c|c|c|c|c|c|c|c|}
\hline \multirow[b]{4}{*}{ Comparison Figure Type } & \multicolumn{12}{|c|}{ Wing Position } \\
\hline & \multicolumn{6}{|c|}{ 50-mm Test Line } & \multicolumn{6}{|c|}{ 100-mm Test Line } \\
\hline & \multicolumn{2}{|c|}{ Both } & \multicolumn{2}{|c|}{ Left } & \multicolumn{2}{|c|}{ Right } & \multicolumn{2}{|c|}{ Both } & \multicolumn{2}{|c|}{ Left } & \multicolumn{2}{|c|}{ Right } \\
\hline & $M$ & $S E$ & $M$ & $\overline{S E}$ & $\bar{M}$ & $\overline{S E}$ & $M$ & $S E$ & $M$ & $\overline{S E}$ & $\bar{M}$ & $\overline{S E}$ \\
\hline Acute angle & 5.21 & 0.82 & 1.72 & 0.80 & 2.06 & 0.55 & 14.71 & 1.18 & 7.60 & 1.29 & 5.56 & 0.90 \\
\hline Obtuse angle & -10.33 & 0.61 & -6.38 & 0.54 & -5.72 & 0.48 & -15.96 & 1.72 & -8.95 & 1.19 & -9.51 & 1.09 \\
\hline
\end{tabular}

ject's task was to indicate the apparent midpoint of each segment. Obviously, if the illusory effect of the angle on the surrounding spatial area decreases systematically as a function of the distance from the angle, systematic midpoint errors in the direction of compensating for the length illusion should occur-the apparent midpoint of each extent should be displaced toward the angle end in the obtuse-angle figure (Figure 4A) and away from the angle in the acute-angle figure (Figure 4B), with greater midpoint errors for the angle than for the no-angle extents. Conversely, accurate midpoint judgments of both extents imply a uniform illusory effect of the angle over the entire length of the shaft.

\section{Method}

The two sets of test stimuli of five stimuli each consisted of a plain horizontal line-the control line-and four single-wing Müller-Lyer stimuli formed from the factorial combination of figure (acute and obtuse angles) and wing position (left and right ends of the shaft). The lengths of the horizontal line of the figures were $50 \mathrm{~mm}$ for one set and $100 \mathrm{~mm}$ for the other, and each stimulus had a 5-mm-long vertical line bisecting the shaft into two physically equal extents. Each stimulus was centered on a sheet of white paper $21 \times 29.5 \mathrm{~cm}$. The dimensions of the stimuli were identical to those of Experiment 1. The stimulus sheet was placed on the surface of a desk, which was covered by white cardboard, with the horizontal line of the stimulus parallel to and $30 \mathrm{~cm}$ away from the edge of the desk adjacent to the subject.

The subject indicated, by drawing a small vertical mark, the apparent midpoint of each the two extents in the figure. Half of the subjects first indicated the midpoint of the left extent followed by the right extent, with the order being reversed for the other half. The two midpoint judgments for each figure were completed before proceeding to the next figure. The 10 stimuli were presented twice; all the stimuli were presented once before any were presented a second time. For each presentation, the 50 - and $100-\mathrm{mm}$-long stimuli were presented in separate blocks of trials, with half the subjects first given the $100-\mathrm{mm}$-long stimulus set and the other half the $50-\mathrm{mm}$ long stimulus set. The presentation of the 5 stimuli in each block was randomized across subjects. There were 16 subjects, none of whom had participated in the previous experiments.

\section{Results}

The distances between the vertical line bisector and the subject's midpoint marks of the two extents of the horizontal line were measured to the nearest $0.5 \mathrm{~mm}$. The measurements for the two presentations of each figure were averaged to yield two scores for each figure, one for the left and one for the right extent. Accurate judgments correspond to 12.5 and $25 \mathrm{~mm}$ for the 50 - and $100-\mathrm{mm}$ long figures, respectively. The means and standard errors, in parentheses, of the midpoint judgments of the left and right extents of the $50-\mathrm{mm}$ control line were $12.4(0.11)$ and $12.1(0.18) \mathrm{mm}$, respectively, and the corresponding means for the 100-mm control line were $24.7(0.19)$ and $24.6(0.28) \mathrm{mm}$. These judgments provided an estimate of each subject's PSE, and each subject's judgments of the Müller-Lyer figures were converted to deviations from PSE by subtracting the PSE from the judgments of the Müller-Lyer stimuli.

The results of the preliminary 2 (figure) $\times 2$ (wing position) $\times 2$ (left/right extents) repeated measures ANOVA performed separately on the $50-$ and $100-\mathrm{mm}$ data sets showed that neither the main effect of wing position nor the interactions involving the wing position factor were significant. The data, therefore, were combined across the wing position variable, and the results are shown in Figure 5 as a function of figure (acute or obtuse angle) and type of extent (angle or no angle). Positive and negative deviation scores indicate that, relative to the PSE, the bisection mark of the extent was drawn closer to and further away from the angle.

The results of $t$ tests showed that the means of the angle extents were significantly greater than zero for both the 50 - and the $100-\mathrm{mm}$ obtuse-angle conditions $[t(15)=5.06$ and $4.84, p<.05$, respectively] and significantly smaller than zero for both the 50 - and the $100-\mathrm{mm}$ acute-angle conditions $[t(15)=6.25$ and $9.49, p<.05$, respectively]. None of the means of the no-angle extents was significantly different from zero $[t(15)=1.20,1.19,1.34$, and $.99, p>.05]$.

The signs of all the acute-angle deviation scores were reversed to produce scores on the same scale as the

A

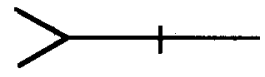

B

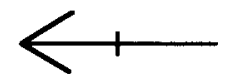

Figure 4. The test figures used in Experiment 3. The vertical line demarcated the shafts into two equally long segments. 
A

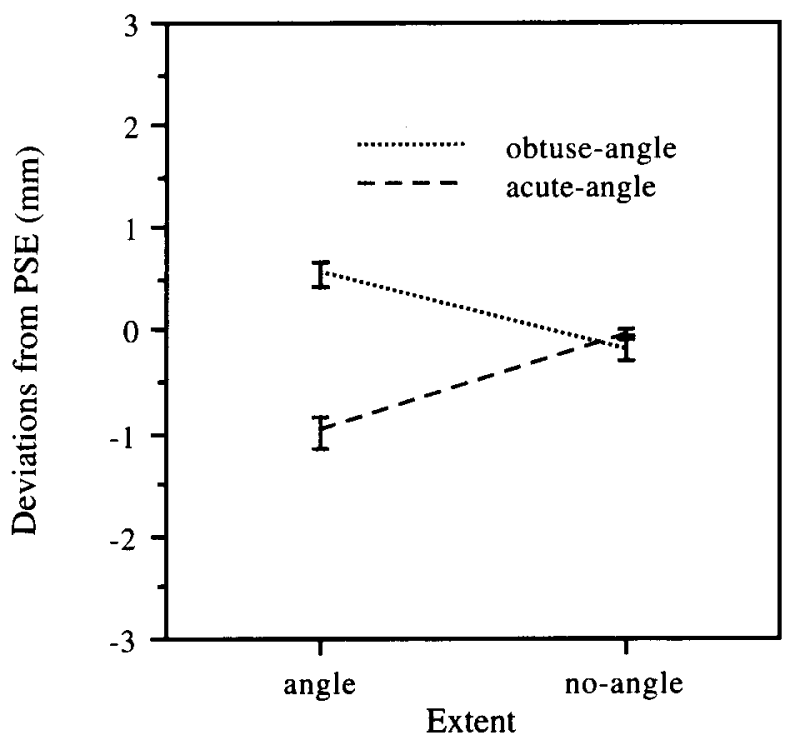

B

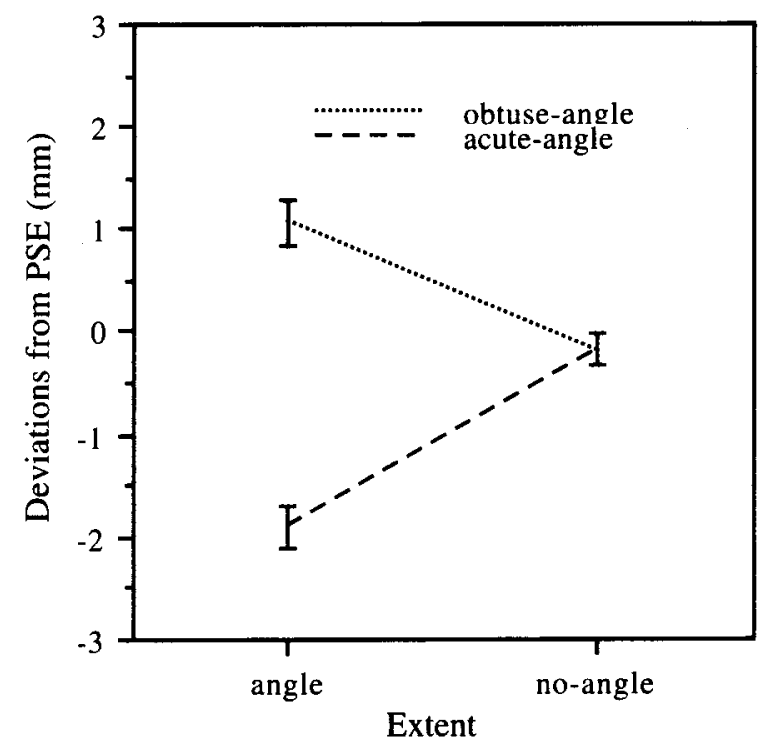

Figure 5. Experiment 3: Means in millimeters of the apparent midpoint errors, expressed as deviations from the point of subjective equality (PSE), for each of the two physically equal long segments of the Müllex-Lyer shaft as a function of type of figure (acute angle or obtuse angle) and type of extent (angle, no angle). Panels $A$ and $B$ are the data sets of the 50- and 100-mm-long figure conditions, respectively. Vertical bars indicate $1 S E$.

obtuse-angle scores. The 2 (figure) $\times 2$ (extent) repeated measures ANOVA performed on the $50-\mathrm{mm}$ scaled data set yielded a significant main effect of figure $[F(1,15)=$ $10.45, p<.05]$, indicating that the mean error was greater for the acute- than for the obtuse-angle conditions $(M=$
0.52 and $0.19 \mathrm{~mm}$, respectively), and a significant main effect of extent $[F(1,15)=46.68, p<.05]$, reflecting the greater for the angle than for the no-angle extent conditions ( $M=0.77$ and $0.01 \mathrm{~mm}$, respectively). The figure $\times$ extent interaction was not significant $[F(1,15)=0.47, p>$ $.05]$; the acute- and obtuse-angle conditions yielded similar patterns of errors across the angle and no-angle extent conditions. The ANOVA performed on the $100-\mathrm{mm}$ data set produced identical outcomes: The figure $\times$ extent interaction was not significant $[F(1,15)=0.87, p>.05]$, the error averaged across the extent factor was greater $[F(1,15)=24.13, p<.05]$ for the acute- than for the obtuse-angle conditions ( $M=1.04$ and $0.45 \mathrm{~mm}$, respectively), and the error averaged across the figure factor was greater $[F(1,15)=64.56, p<.05]$ for the angle than for the no-angle extent conditions $(M=1.49$ and $0.0 \mathrm{~mm}$, respectively).

\section{Discussion}

The results indicate a general similarity in the pattern of bisection errors across the acute- and obtuse-angle Müller-Lyer figures and, thus, are inconsistent with claims that acute and obtuse angles have different scaling effects on the perceived lengths of the shafts. For both Müller-Lyer forms, there were substantial errors for the segment adjacent to the angle and negligible errors for the segment distant from the angle. A discussion of these findings is postponed to a later section. For the moment, two aspects of the present findings require comment.

First, the significant main effect of figure in the ANOVA performed on the scaled data scores indicates greater bisection errors for the acute- than for the obtuse-angle figures. This outcome, however, does not necessarily imply differences in the scaling effects (i.e., the spatial range of influence) of the acute and obtuse angles; the effects may differ in absolute magnitude but not in their spatial ranges. The crucial finding is the nonsignificant figure $\times$ extent interaction, which indicates an equivalence of the acuteand obtuse-angle illusion conditions in the pattern of bisection errors; for both conditions, there were substantial illusory effects for the angle extent and negligible effects for the no-angle extent.

Second, the negligible bisection errors of the no-angle extent conditions for both the acute- and the obtuse-angle forms appear to be inconsistent with Warren and Bashford's (1977) claims of a differential scaling effect of the angle components. However, Experiment 3 differed from Warren and Bashford's methodology in one, perhaps important, respect: Whereas they had subjects bisect the shaft and, then, the resulting two apparently equal segments, to produce subjectively equal quarters, in Experiment 3 , the vertical line bisector produced two subjectively unequal segments, as is evident from Figure $4 .^{3}$ Arguably, the subjectively unequal segments produced by the bisector reduced the tendency toward a graded illusory effect of the angles. Furthermore, and for whatever reasons, the presence of the bisector may have minimized the contextual influences of the angles on the apparent 
A

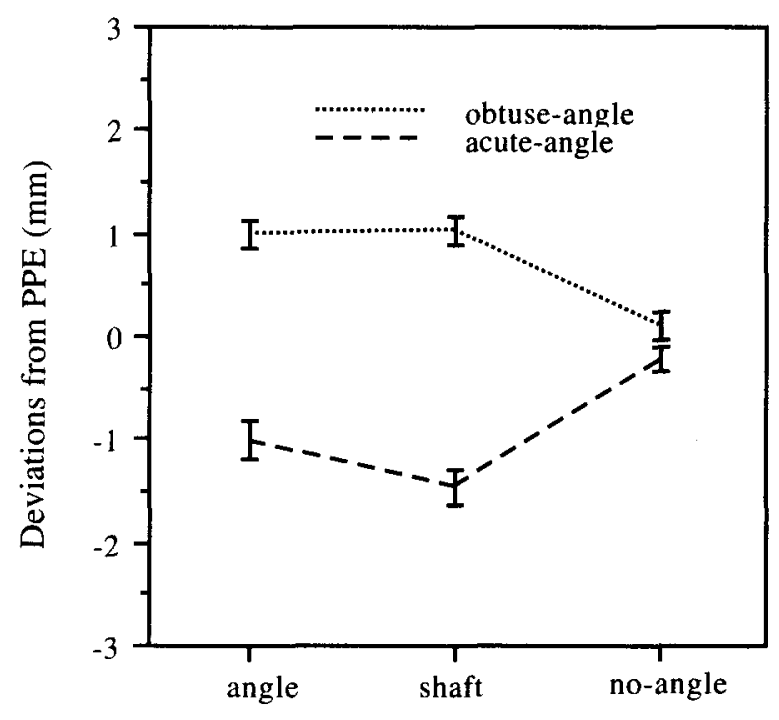

B

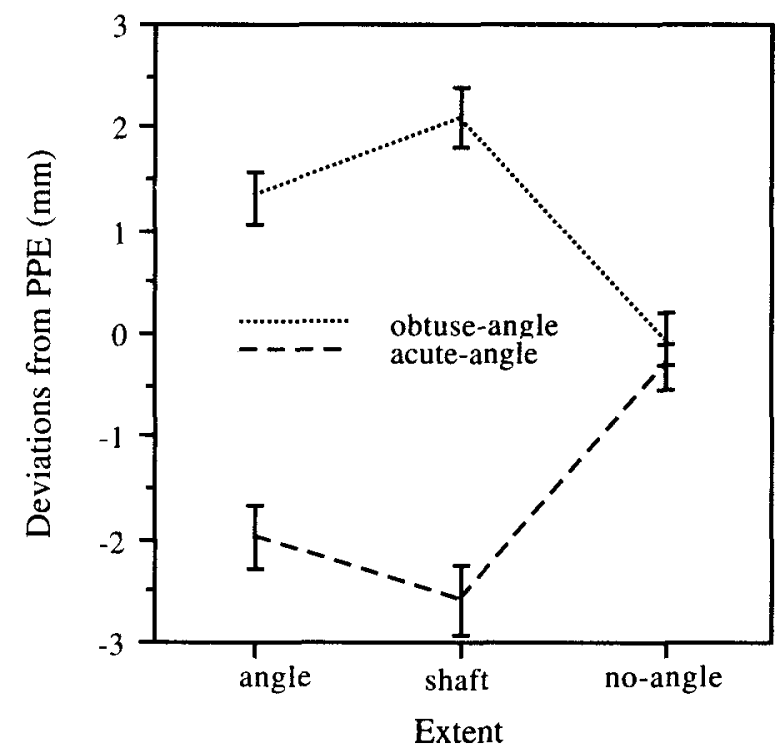

Figure 6. Experiment 4: Means in millimeters of the apparent midpoint errors, expressed as deviations from the point of physical equality (PPE), as a function of type of figure (acute angle or obtuse angle) and type of extent (shaft, angle, and no-angle segments). Panels $A$ and $B$ are the data sets of the 50- and $100-\mathrm{mm}-$ long figure conditions, respectively. Vertical bars indicate $1 S E$.

lengths of the no-angle segments. The present findings, therefore, are not necessarily inconsistent with those of Warren and Bashford; the inconsistency may reflect the different processes elicited by the presence in Experiment 3 and the absence in their experiments of the vertical line bisector. This possibility was examined in Experiment 4 , which used Warren and Bashford's procedure of fractionating the Müller-Lyer figures into subjectively equal quarters. In addition, it assessed the scaling effects of the angles in the conventional (i.e., two-wing) MüllerLyer figures.

\section{EXPERIMENT 4}

\section{Method}

The stimuli were eight single-wing and four two-wing (conventional) Müller-Lyer figures. The single-wing figures ( 2 lengths $X$ 2 wing positions $\times 2$ figures) were identical to those used in Experiment 3 (see Figure 3), except that the shafts did not contain the vertical line bisector. The conventional figures were formed from the factorial combination of figure (acute or obtuse angle) and shaft length $(50$ or $100 \mathrm{~mm})$. The subjects first bisected the shaft and then bisected each of the two resulting segments, to produce four apparently equal segments. The subjects practiced the fractionation task on 30-, 75-, and 120-mm-long plain horizontal lines.

Unlike Experiment 3, each figure was presented only once for judgment. The single-wing figures were presented first, with the 50- and 100-mm-long stimuli being presented in separate blocks of trials. Half of the subjects were first given the $100-\mathrm{mm}$-long stimulus set, and the other half the 50-mm-long stimulus set, with the presentation of the four stimuli in each block randomized across subjects. The presentation of the four conventional figures was randomized across subjects. In all other respects, the procedure was identical to that in Experiment 3. The subjects were 27 volunteer undergraduate students, none of whom had participated in the previous experiments.

\section{Results}

Single-wing figures. The distances of the bisection marks from the angle end of the shaft were measured to the nearest $0.5 \mathrm{~mm}$. The first judgment, which produced two subjectively equal extents (angle, no angle), were converted to deviations from the point of physical equality (PPE) -25 or $50 \mathrm{~mm}$ for the $50-$ and $100-\mathrm{mm}$ stimuli, respectively. The deviation scores were assigned positive or negative values if the mark was placed toward or away from the angle end of the shaft, respectively. The PPEs for the apparent midpoints of the angle and no-angle extents formed by the first bisection mark were referenced to the actual lengths of the extents. For example, if a subject's first bisection judgment of a $100-\mathrm{mm}$ figure was $44 \mathrm{~mm}$ from the angle end, then (by definition) the actual lengths of the apparently equal angle and no-angle extents were 44 and $56 \mathrm{~mm}$, respectively, and the resulting PPEs were $22 \mathrm{~mm}(44 / 2)$ and $72 \mathrm{~mm}(56 / 2+44)$. Thus, bisection marks of the angle and no-angle extents that were drawn 20 and $73 \mathrm{~mm}$, respectively, from the angle end represent deviation scores of $+2(22-20)$ and $-1(72-$ 73) $\mathrm{mm}$, respectively.

Neither the main effect of wing position nor the interactions involving wing position were significant in the ANOVA performed on the 50 - and $100-\mathrm{mm}$ data sets. The scores, therefore, were combined across the wing position variable, and the results are shown in Figure 6.

The results of the $t$ tests indicated that the means of the deviation scores of the shaft and of the angle extent conditions were significantly smaller than zero for both the 50 - and the $100-\mathrm{mm}$ acute-angle conditions and greater 


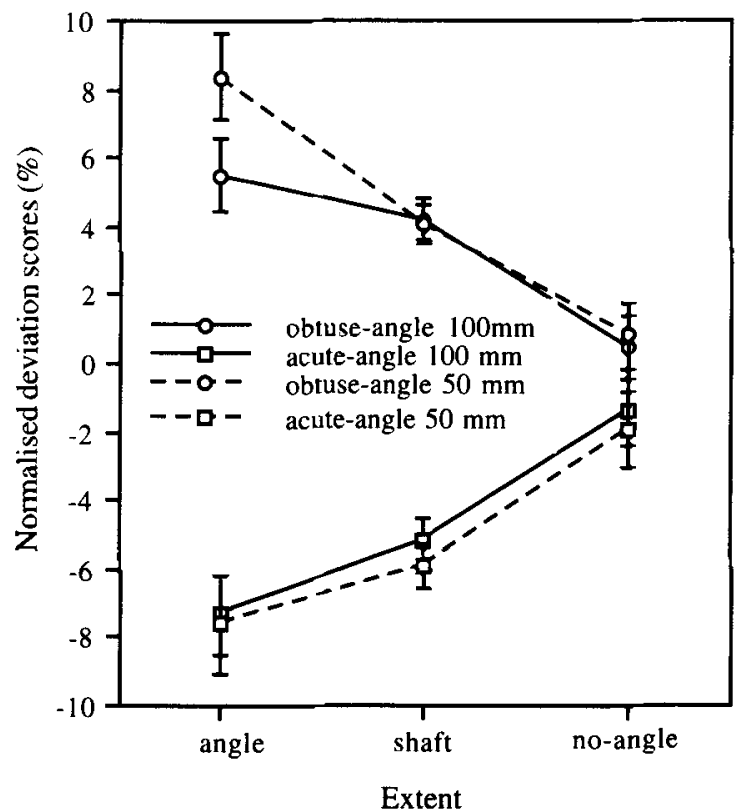

Figure 7. Experiment 4: Means and standard errors of the normalized deviation scores (error/length $X 100$ ) as a function of figure (acute angle or obtuse angle), type of extent (shaft, angle, and no-angle segments), and length of shaft $(50 \mathrm{or} 100 \mathrm{~mm})$. Vertical bars indicate $1 S E$.

than zero for both the 50 - and the $100-\mathrm{mm}$ obtuse-angle conditions [all $t(26) \mathrm{s}>5.22, p<.05]$. The means of the no-angle extents were not significantly different from zero for the 50- and 100-mm obtuse-angle conditions and the 50 -mm acute-angle condition $[t(26)=0.86,0.79$, and 1.44 , $p>.05$, respectively]; the mean of the $50 \mathrm{~mm}$ acute-angle condition was marginally nonsignificant $[t(26)=1.64, p=$ $.06]$.

For reasons that will be evident later, paired $t$ tests compared the means of the angle extent and shaft conditions. The comparison was not significant for the 50 -mm obtuseangle condition $[t(26)=0.21, p<.05]$ and was marginally significant for the 50-mm acute-angle $[t(26)=2.02, p=$ $.027]$, the $100-\mathrm{mm}$ obtuse-angle $[t(26)=1.72, p=.048]$ and the $100-\mathrm{mm}$ acute-angle $[t(26)=1.75, p=.046]$ conditions.

As for Experiment 3, the signs of the acute-angle deviation scores were reversed to produce scores on the same scale as the obtuse-angle scores. The 2 (figure) $\times 3$ (extent) repeated measures ANOVA performed on the 50 $\mathrm{mm}$ scaled data yielded a significant main effect of extent $[F(2,52)=36.73, p<.05]$; averaged across the figure factor, the means were $1.25,0.99$, and $0.16 \mathrm{~mm}$ for the shaft, angle, and no-angle extents, respectively. Neither the main effect of figure $(M=0.90$ and $0.71 \mathrm{~mm}$ for the acute- and obtuse-angle conditions, respectively) nor the figure $\times$ extent interaction was significant $[F(1,26)=$ 2.11 and $F(2,52)=0.86$, respectively, $p>.051$. The
ANOVA performed on the $100-\mathrm{mm}$ data set yielded a significant main effect of extent $[F(2,52)=37.76, p<$ $.05]$; averaged across the figure factor, the means of the shaft, angle, and no-angle extents were $2.34,1.65$, and $0.13 \mathrm{~mm}$, respectively. The main effect of figure was significant $[F(2,52)=14.00, p<.05]$; acute-angle errors were greater than the obtuse-angle errors $(M=1.63$ and $1.17 \mathrm{~mm}$, respectively). The extent $\times$ figure interaction was not significant $[F(2,52)=0.08, p>.05]$.

The data in Figure 6 suggest a similarity in the pattern of errors of the 50 - and $100-\mathrm{mm}$ data sets. To determine whether the similarity is scale invariant, the deviation scores were normalized by dividing each bisection score by the relevant length of the bisected length, either the actual length of the shaft or the measured lengths of the angle and no-angle extents resulting from the first bisection mark. The means and standard errors of the normalized data, expressed as percentage scores [(bisection error)/ length $\times 100$ ], are presented in Figure 7, which clearly show the similarity of the 50 - and the $100-\mathrm{mm}$ data sets.

Two-wing (conventional) figures. The bisection judgments of the shaft were accurate: None of the means of the 50- and 100-mm conditions ( $M=0.02,0.11,0.06$, and $0.15 \mathrm{~mm}$ ) was significantly different from the PPE (25 and $100 \mathrm{~mm}$ for the 50 - and $100-\mathrm{mm}$ figures, respectively). For the 100-mm data set, the means and standard errors (in parentheses) of the bisection judgments of the left and right segments resulting from the first bisection mark, which divided the shaft into subjectively equal segments, were $-1.92(0.35)$ and $-1.81(0.36) \mathrm{mm}$ for the acute-angle figure, and $1.44(0.29)$ and $1.31(0.26) \mathrm{mm}$ for the obtuse-angle figure, respectively. All the means were significantly different from zero [ $t(26) \mathrm{s}>5.06, p<.05$ ] and in the direction of the illusion; the apparent midpoints of both the left and the right segments were displaced toward and away from the angle end of the segment for the obtuse- and acute-angle figures, respectively. The corresponding means for the $50-\mathrm{mm}$ figures were -0.82 $(0.17)$ and $-1.32(0.20) \mathrm{mm}$ for the acute-angle figure and $0.85(0.19)$ and $0.96(0.20) \mathrm{mm}$ for the obtuse-angle figure; all the means were significantly different from zero $[t(26) s>4.46, p<.05]$ and in the expected direction.

\section{Discussion}

The results of the one-wing conditions are consistent with the outcomes of Experiment 3. In both experiments, the acute- and obtuse-angle figures yielded similar patterns of bisection errors: Judgments of the apparent midpoint of the angle extent, but not of the no-angle extent, showed systematic errors in the direction of the illusion. It seems unlikely, therefore, that the outcomes of Experiment 3 were influenced by the vertical line bisector in the figures. The data of the conventional figure conditions also indicate a similarity in the scaling effects of the acute- and obtuse-angle components, a result that stands 
in marked contrast to Warren and Bashford's (1977) findings. Finally, the normalized data generally indicate an equivalence in the proportional magnitudes of illusory effects across the 50- and 100-mm figure conditions, suggesting the scale independence of whatever mechanisms are responsible for the bisection errors.

Although not directly relevant to the present investigation, an intriguing aspect of the results of Experiments 3 and 4 deserves mentioning. Specifically, unlike the usual finding (e.g., Christie, 1975; Day \& Dickinson, 1976; Predebon, 1992; see also Experiments 1 and 2) of greater length illusion magnitudes for obtuse-angle Müller-Lyer figures, the magnitudes of the bisection errors for the acute-angle figures were either greater than (Experiments 3 and 4), or similar to (Experiment 4) those of the corresponding obtuse-angle conditions. Additional work, which was beyond the scope of the present investigation, is required to identify the basis for this difference (e.g., it may reflect different sources of stimulus information accessed by the two tasks). In any case, this finding does not affect the major conclusion drawn from the results of Experiments 3 and 4-namely, the similarity in the pattern of bisection errors across the acute- and obtuse-angle forms of the Müller-Lyer illusion.

\section{GENERAL DISCUSSION}

The aims of the four experiments described here were to evaluate the effect of reducing the conventional Müller-Lyer figures to their single-wing versions on the magnitudes of the acute- and obtuse-angle length illusions (Experiments 1 and 2) and to assess whether the acuteand obtuse-angle components have similar or different scaling effects on the apparent length of the shaft (Experiments 3 and 4).

The results of Experiments 1 and 2 are consistent with the conclusions drawn from the results of Day and Dickinson's (1976) and Predebon's (1996) studies but are inconsistent with those of Warren and Bashford (1977), which show little reduction in illusion magnitude for the acute-angle figures. They also appear to be inconsistent with Redding and Hawley's (1993) claim of a greater reduction occurring for the obtuse- than for the acute-angle illusion forms, although, as was mentioned previously, these investigators may have overstated their findings.

As indexed by judgments of the midpoints of the extents in the one-wing figures, the results of Experiments 3 and 4 demonstrate the similarity of the scaling effects of the acute- and obtuse-angle components. For neither angle component did the illusory effect operate uniformly across the entire length of the shaft: Bisection errors occurred for judgments of the midpoint of the shaft (Experiment 4) and of the segment of the shaft adjacent to the angle (Experiments 3 and 4) but not of the segment of the shaft distant from the angle (Experiments 3 and 4). Furthermore, for both of the conventional Müller-Lyer forms, the angles induced systematic bisection errors. These findings, especially the latter, are inconsistent with Warren and Bashford's (1977) major claim that, whereas the acute-angle scaling effect is restricted mainly to the region of the shaft adjacent to the wings, the obtuse-angle scaling effect operates more or less uniformly over the entire length of the shaft. Instead, they are consistent with Morinaga's work, as described by Oyama (1960): "Morinaga (1941) fractionated the main line into seven physically equal parts and observed apparent length of each part. All parts but the parts directly connected to the oblique lines appeared equal. This means that illusion occurs only in adjacent area of the angles" (p. 8). ${ }^{4}$

The importance of Warren and Bashford's (1977) work, and of its apparent confirmation by Redding and Hawley (1993), resided in its challenge to virtually all theories of the Müller-Lyer illusion. The illusion magnitude results of Experiments 1 and 2, however, are consistent with most theories of the illusion and with the view that the two illusion forms represent fundamentally similar illusion types (Predebon, 1996). Nevertheless, the bisection results of Experiments 3 and 4, together with those of Morinaga (Oyama, 1960), have implications for certain length attribute theories of the illusion, such as assimilation theories, which emphasize interactions in the length domain without reference to the relative positions of the contextual and focal extents. For example, Brigell et al.'s (1977) neurophysiological theory of size illusions assumes that the apparent length of a focal extent presented alone (e.g., the shaft of the Müller-Lyer form) is determined by a central measure of the output of the population of length-sensitive neural units, each of which responds to a limited range of lengths. Incorporating the focal extent in a context, such as placing wings at the end of the shaft, alters the distribution of neural activity in response to the simultaneous presentation of the focal and contextual stimuli, with the judgment of the shaft's length weighted by the response to the shaft presented alone. However, without invoking additional position-specific mechanisms, distribution shift models of this kind cannot explain why the influence of the wing components is confined mainly to the region of the shaft adjacent to the angle.

Finally, two general issues require comment. The first concerns the relationship between the length-matching and bisection findings. Arguably, given the widespread belief (see Coren \& Girgus, 1978, for review) of multiple causation of the Müller-Lyer illusion, some of the determinants (e.g., perceptual assimilation mechanisms) of the illusion may operate uniformly across the entire length of the shaft, whereas others (e.g., position-encoding mechanisms) are restricted to the region of the angle components. Uniform illusory effects, of course, cannot be measured (i.e., detected) by the bisection task. Thus, the bisection errors found in Experiments 3 and 4 necessarily index only the contribution of those mechanisms producing localized illusory effects, whereas the length-matching er- 
rors of Experiments 1 and 2 reflect the combined contribution of all the illusion-inducing mechanisms, including those eliciting uniform effects, responsible for the metric distortion of the shaft's length.

The second issue relates to the nature of the angle's localized illusory effects, as indicated by the results of Experiments 3 and 4 . One possibility-the gradient hypothesis - is that the angle's influence on the shaft decreases as a function of the distance between points on the shaft and the angle. This hypothesis is not considered further here, since the gradient of the illusory effects remains to be specified. The other possibility - the local hypothesis-is suggested by the position displacement theories of the Müller-Lyer illusion (Chiang, 1968; Morgan et al., 1990). As was noted previously, these theories attribute the Müller-Lyer illusion to the illusory displacement of the angle's vertex, rather than to a scaling effect of the angle on the shaft's apparent length. The local hypothesis therefore predicts, as is the case, negligible bisection errors for the no-angle extent conditions of Experiments 3 and 4 ; since neither endpoint of the extents is defined by an angle, the locations of the endpoints will be encoded correctly, resulting in accurate midpoint judgments.

The local hypothesis, however, appears to be contradicted by the results of the shaft and angle extent conditions of Experiment 4 . The hypothesis predicts identical bisection error magnitudes for the shaft and the angle extent conditions; whereas the locations of the no-angle, or free, end of the extents (i.e., the open end of the shaft for the shaft condition, and the mark bisecting the shaft for the angle extent condition) are perceived accurately, the location of the angle end of the extents is perceptually displaced by the same amount in the shaft and the angle extent conditions. The results of the relevant $t$ tests (see Experiment 4 ) showed that only for the 50 -mm obtuse-angle condition was there a clear equivalence in the magnitudes of the bisection errors of the shaft and the angle extent; for the other conditions, the errors were greater for the shaft than for the angle extent. It would be premature to dismiss the local hypothesis on this ground alone, however. For one, independent evidence, preferably obtained with a methodology different from that employed in the present investigation, is required to confirm the results of Experiment 4, especially since the difference between the shaft angle extent errors was either not significant or marginally significant. For another, the hypothesis is appealing in its simplicity, especially in its straightforward explanation of Morinaga's findings, described above. According to this hypothesis, the locations of the endpoints of the five segments of the shaft not directly connected to the angles are encoded correctly, with the result that these segments should appear equal in length, whereas the remaining two segments (one at each end of the shaft) directly connected to the angle should appear different in length from the other five segments as a consequence of the illusory displacement of the locations of the angle end of the segments.

\section{REFERENCES}

Brigell, M., Uhlarik, J., \& Goldhorn, P. (1977). Contextual influences on judgments of linear extent. Journal of Experimental Psychology: Human Perception \& Performance, 3, 105-118.

Chiang, C. (1968). A new theory to explain geometrical illusions produced by crossing lines. Perception \& Psychophysics, 3, 174-176.

Christie, P. (1975). Asymmetry in the Müller-Lyer illusion: Artefact or genuine effect? Perception, 4, 453-457.

COREN, S., \& Girgus, J. S. (1978). Seeing is deceiving: The psychology of visual illusions. Hillsdale, NJ: Erlbaum.

DAY, R. H., \& Dickinson, R. G. (1976). Apparent length of the arms of acute and obtuse angles and the components of the Müller-Lyer illusion. Australian Journal of Psychology, 26, 49-59.

Grllam, B. (1998). Illusions at century's end. In J. Hochberg (Ed.), Perception and cognition at century's end (pp. 95-136). San Diego: Academic Press.

GrnsBurG, A. P. (1984). Visual form perception based on biological filtering. In L. Spillmann \& B. R. Wooten (Eds.), Sensory experience, adaptation, and perception: Festschrift for Ivo Kohler (pp. 53-72). Hillsdale, NJ: Erlbaum.

Glennerster, A., \& Rogers, B. (1993). New depth to the Müller-Lyer illusion. Perception, 22, 691-704.

GREGORY, R. (1963). Distortions of visual space as inappropriate constancy scaling. Nature, 199, 678-680.

Morgan, M. J., Hole, G. J., \& Glennerster, A. (1990). Biases and sensitivities in geometrical illusions. Vision Research, 30, 1793-1810.

Müller-LyER, F. C. (1889). Optische-Urteilstäuschungen. Archiv für Anatomie \& Physiologie: Physiologische Abteilung, 2(Suppl.), 263270.

OYama, T. (1960). Japanese studies on the so-called geometrical-optical illusions. Psychologica, 3, 7-20.

Predebon, J. (1992). Framing effects and the reversed Müller-Lyer illusion. Perception \& Psychophysics, 52, 307-314.

Predebon, J. (1994). The reversed Müller-Lyer illusion in conventional and in wing-amputated Müller-Lyer figures. Psychological Research, 56, 217-223.

Predebon, J. (1996). The role of the angle components in the wings-in and wings-out forms of the Müller-Lyer illusion. Perception, 25, 773-781.

Pressey, A. W., \& Pressey, C. A. (1992). Attentive fields are related to focal and contextual features: A study of Müller-Lyer distortions. Perception \& Psychophysics, 51, 423-436.

RedDING, G. M., \& HaWLEY, E. (1993). Length illusion in fractional Müller-Lyer stimuli: An object-perception approach. Perception, 22, 819-828.

Restle, F., \& Decker, J. (1977). Size of the Mueller-Lyer illusion as a function of its dimensions: Theory and data. Perception \& Psychophysics, 21, 489-503.

RoBINSON, J. O. (1972). The psychology of visual illusion. London: Hutchinson.

Stuart, G. W., Day, R. H., \& Dickinson, R. G. (1984). Müller-Lyer: Illusion of size or position? Quarterly Journal of Experimental Psychology, 36A, 663-672.

WARREN, R. M. (1976). Auditory illusions and perceptual processes. In N. J. Lass (Ed.), Contemporary issues in experimental phonetics (pp. 389-417). New York: Academic Press.

WARREN, R. M., \& BASHFoRd, J. A. (1977). Müller-Lyer illusions: Their origin in processes facilitating object recognition. Perception, 6, 615626.

\section{NOTES}

1. Warren and Bashford (1977) used the logistically expensive betweensubjects design, in which separate groups of subjects $(N=30$ in each group) were assigned to each display, a design that also prohibited in- 
cluding a within-subjects control condition. For these reasons, I modeled Experiment 1 on Redding and Hawley's (1993) design.

2. Previous studies (for a review, see Robinson, 1972) indicate that maximum illusory effects occur for a wing length to shaft length ratio of approximately $0.3: 1$, at least for the conventional (i.e., two-wings) Müller-Lyer forms.

3. This was confirmed by the results of a supplementary study. The stimuli were identical to those in the main experiment, except that they did not contain the vertical bisecting line. The subjects $(N=14)$ penciled a mark at the shaft's apparent midpoint. The means and standard errors (in parentheses) of the midpoint deviation scores were $1.30(0.12)$ and $-0.91(0.13)$ for the $50-\mathrm{mm}$ obtuse- and acute-angle figures, respectively, and the corresponding results for the $100-\mathrm{mm}$-long figures were 2.05 $(0.33)$ and $-1.79(0.33) \mathrm{mm}$; all the means were significantly different from zero $[t(13) \mathrm{s}>5.41, p<.05]$ and in the direction of the illusion.
4. As was described by Oyama (1960), Morinaga also replicated Yanagisawa's "reverse" Müller-Lyer findings - namely, the decrease of the obtuse-angle illusion and the reversal of the acute-angle illusion from under- to overestimation as a function of the distance or gap between the ends of the shaft and the vertices of the wing components (see Predebon, 1994). Given Morinaga's awareness of this fundamental difference between the two illusion forms, it seems reasonable to assume that he investigated the effect of fractionating the shaft component on both the acute- and the obtuse-angle forms. Consequently, I interpret Oyama's summary of Morinaga's results as referring to both forms of the illusion.

(Manuscript received August 4, 1998; revision accepted for publication June 28, 1999.) 